\title{
Visión de futuro de la Sociedad Chilena de Enfermedades Respiratorias**
}

\author{
FRANCISCO ARANCIBIA H.*
}

\section{Chilean Society of Respiratory diseases' foresight}

An unavoidable role of scientific associations in $21^{\text {st }}$ century is to promote, to stimulate, and to have influence on the social, professional, and scientific environment of their countries. To achieve these objectives the Chilean Society of Respiratory Diseases should develop or strengthen on the following three bases: education, research and involvement of their members. Besides, we cannot stop our fight against smoking, which remains the leading cause of preventable illness and death in Chile and the world. Finally, it is necessary to promote members participation, to create a strategic plan and to obtain financial resources to get this vision become a reality.

Key words: Future; Scientific association.

\section{Resumen}

En el contexto actual, las sociedades cientificas tienen el ineludible reto de impulsar, estimular e influir en el entorno social, profesional y científico del país. Para lograr estos objetivos la Sociedad Chilena de Enfermedades Respiratorias debe desarrollar o consolidar los siguientes tres pilares: la educación, la investigación y la participación. También, no podemos cesar nuestra lucha contra el tabaquismo, que continúa siendo la principal causa de enfermedades y muertes prevenibles en Chile y el mundo. Finalmente, para que esta visión de futuro llegue a ser realidad es necesario promover la participación de sus miembros, crear un plan estratégico y obtener recursos financieros.

Palabras clave: Visión de futuro, Sociedad científica.

El futuro proviene del latín futūrus, y se define como aquello que está por venir. En el contexto actual, las sociedades científicas tienen el ineludible reto de impulsar, estimular e influir en el entorno social, profesional y científico, con el fin de contribuir al máximo a facilitar la adaptación, perfeccionamiento continuo e innovación permanente de las especialidades y de quienes las desarrollan y representan.

Estimo que los tres pilares fundamentales que deben ser desarrollados por la Sociedad Chilena de Enfermedades Respiratorias (SER-Chile) deben ser: 1) la educación; 2) la investigación y 3) la participación.
Sin duda, que una de las actividades importantes y permanentes de la SER-Chile es la $\boldsymbol{e d u}$ cación continua a través de los cursos, talleres, jornadas y congresos que ofrece la sociedad. Por otro lado, los cursos e-learning son una herramienta tecnológica educativa cada vez más importante. Este nuevo concepto educativo es una revolucionaria modalidad de capacitación que posibilitó Internet, y que hoy se posiciona como la forma de capacitación predominante en el futuro. Los cursos e-learning tienen varias ventajas que debemos considerar, entre las que se encuentran: reducción de costos, rapidez y agilidad de las comunicaciones, acceso al contenido desde

\footnotetext{
* Vicepresidente, Sociedad Chilena de Enfermedades Respiratorias. Jefe Servicio de Medicina, Instituto Nacional del Tórax Profesor Agregado, Facultad de Medicina, Universidad de Chile.
}

\footnotetext{
**Texto basado en la conferencia pronunciada en las $34^{\text {as }}$ Jornadas de Otoño de la Sociedad Chilena de Enfermedades Respiratorias, Olmué, V Región. 28 de marzo, 2015.
} 
cualquier conexión a Internet, y flexibilidad de la agenda.

En ese sentido la SER-Chile debe potenciar esta actividad, es prioritario el perfeccionamiento continuo con e-learning y aprovechar la tecnología actual disponible con la idea de llegar a todos lo rincones de Chile y también de América Latina, con un costo mínimo para los socios.

Todas estas actividades deberían ser válidas, como capacitación respaldadas por nuestra Sociedad para la certificación de la especialidad. Es más, la Sociedad debiera desarrollar una estrategia de preparación para los exámenes de certificación de la especialidad, como ya lo hacen otras sociedades como la Sociedad de Psiquiatría con cursos on-line.

Para aquellos que se van a re-certificar próximamente, la Sociedad debería otorgar créditos como lo hacen otras sociedades internacionales a cursos, jornadas, congresos, talleres, etc., que permiten conocer el estado de vigencia de nuestros especialistas y que sean reconocidas por el Ministerio de Salud y la Corporación Nacional Autónoma de Certificación de Especialidades Médicas (CONACEM).

Es necesaria una asociación estratégica con el Ministerio de Salud y centros de formación universitaria para ofrecer actividades de capacitación, con pasantías nacionales para nuestros socios de las regiones del país. También, profundizar los convenios con sociedades extranjeras para poder optar a pasantías internacionales avaladas por la Sociedad, para nuestros socios que deseen ponerse al día en el manejo clínico-práctico en patología respiratoria.

La Sociedad debe tener una especial preocupación por los becados de la especialidad. En Chile, el número de especialistas broncopulmonares es insuficiente, sobre todo en regiones. Lo dicen las autoridades del Ministerio de Salud y una reciente publicación de Vásquez y colaboradores en la revista Archivos de Bronconeumología, corrobora esta información en la que da cuenta de la tasa de neumólogos en América Latina y el Caribe ${ }^{1}$. Chile, se sitúa con un déficit importante de especialistas, con un índice de 0,7 médicos broncopulmonares por 100.000 habitantes. Estos indicadores se encuentran muy por debajo del estándar (2 por 100.000 habitantes), o la tasa de España o Estados Unidos que tienen 3 por 100.000 habitantes. Esta situación es preocupante, e implica que faltan alrededor de 221 neumólogos para alcanzar el estándar y al ritmo actual de formación de especialistas demoraríamos unos 36 años para alcanzarlo. Creo de máxima importancia que la Sociedad participe en la definición del tipo de especialista broncopul- monar que requiere nuestro país para el siglo XXI. La Sociedad debe involucrarse en la formación de estos futuros especialistas, en conjunto con las universidades y el Ministerio de Salud, privilegiando un currículum por competencias. Atrás quedó, un excelente curso de Fisiopatología que otorgaba la Sociedad a los becados de enfermedades respiratorias del país. Es hora de recuperar esa instancia de docencia y entrega de conocimientos desde la Sociedad a nuestros becados.

Por otro lado, nuestra Revista Chilena de Enfermedades Respiratorias, es el órgano oficial de la Sociedad y posee una larga y prestigiosa historia. La dirigen, con gran profesionalismo y trabajo, el Dr. Manuel Oyarzún y el Dr. Fernando Saldías. La revista debe ser apoyada por todos los miembros de la Sociedad, pero con hechos tangibles como escribir una crónica, una revisión o publicar la investigación realizada, lo cual contribuirá para que nuestra revista pueda ser incorporada al International Scientific Indexing (ISI Thomson Reuters). Un ejemplo a seguir es el camino que ha realizado, la Revista Chilena de Infectología. Ésta, siendo una sociedad más pequeña que la nuestra, desarrolló una estrategia con objetivos claros basado en el trabajo de los comités, que son muy activos y productivos, logrado posicionarse y alcanzar el tan ansiado índice ISI. En la actualidad la Revista Chilena de Infectología tiene un factor de impacto de 0,497. Así también, debemos continuar trabajando en guías temáticas, resumiendo la evidencia actual en distintas patologías, para ser el referente natural en la toma de decisiones.

La Investigación es otro de los pilares que debe sustentar a la Sociedad y debería ser otra de las prioridades. Debemos fomentar y desarrollar la investigación, priorizando los proyectos multidisciplinarios y también los de colaboración internacional, apoyar su financiamiento y formar investigadores en el área. Contamos con un comité científico de alto nivel presidido por la Dra. Patricia Díaz, para esta tarea. La Sociedad debe potenciar el desarrollo de los grupos de investigación del país y aumentar los lazos de trabajo con sociedades nacionales e internacionales como: la Asociación Latinoamericana de Tórax (ALAT), la Sociedad Mexicana de Neumología y la Sociedad Española de Patología Respiratoria (SEPAR), entre otras. En este sentido, hay camino avanzado con convenios con la SEPAR y la ALAT y esperamos avanzar en investigaciones colaborativas. Creemos que los motores de la Sociedad son sus comisiones y es de nuestro interés promover que tengan un rol más activo e influyente en las decisiones tema-país con respecto a salud respiratoria. 
Como Sociedad estamos contribuyendo muy poco al desarrollo de la investigación de nuestro país. En los últimos años, el fondo de financiamiento para la investigación de que dispone la sociedad para sus miembros ha quedado desierto de postulantes o han sido muy pocos quienes han postulado. Es probable que debamos aumentar tanto en montos, como en el número de proyectos de investigación financiados por la Sociedad para estimular a los grupos de investigación, de preferencia colaborativos con regiones y que incluyan investigadores jóvenes.

También es necesario, capacitar a los profesionales de la salud dedicados al área de respiratorio, otorgando herramientas básicas para realizar investigación científica clínica como básica, sobre todo a las generaciones más jóvenes. También, se requiere apoyo de un especialista en bioestadística, tanto en el diseño del estudio como en el análisis de los datos, y en el adiestramiento en el manejo de herramientas estadísticas computacionales. Queremos una SER que colabore activamente en la génesis de conocimiento médico con datos nacionales. Hoy en día tenemos una oportunidad para investigar dado por: 1) la relevancia de las enfermedades respiratorias por su crecimiento en la prevalencia y en el impacto clínico, social y económico y 2) los medios masivos de comunicación y electrónicos nos facilitan la interacción e intercambio entre los investigadores de la región y la obtención de información y su difusión.

Un tercer pilar de la Sociedad y clave es la participación. Debemos fomentar y dar acceso a la participación en la toma de decisiones de la Sociedad. Llama la atención que sólo $1 / 3$ de los socios activos participan de las elecciones de la directiva, que se realiza cada dos años. La votación on-line, debe fomentarse y puede ser una herramienta útil en la mayor participación que puedan tener los miembros de la Sociedad.

También, se requiere una mayor participación de nuestros miembros en los diversos comités de la Sociedad. Cada socio debería pertenecer a 1 ó 2 comités, los cuales deberían ser la fuente de las diversas actividades, consensos, guías, e investigación de la Sociedad. Queremos una Sociedad, acogiendo la experiencia, pero también potenciando a las generaciones más jóvenes, que son la fuente de dinamismo e innovación. Es fundamental, que los jóvenes neumólogos vayan tomando roles en la sociedad, porque en un futuro próximo serán autoridades directivas que guiarán a la Sociedad.

Con respecto a las Regiones, es importante potenciar el trabajo de las filiales y aumentar su participación, comprometiendo desde ya un mayor apoyo desde la directiva central. La participación debe incluir actividades con la comunidad, realizando talleres, vídeos informativos para los pacientes de los Centros de Salud Familiar (CESFAM), educando a los colegios y formando monitores sobre los graves daños que ocasionan por ejemplo el tabaquismo y otras noxas de impacto social.

También, debe continuar la participación activa de la Sociedad con entidades gubernamentales, como el Ministerio de Salud y constituirse en un impulsor de las políticas para el país en salud respiratoria.

Se ha avanzado en la participación con otras sociedades y actualmente está vigente un convenio con la Sociedad Española de Patología Respiratoria que ha dado sus frutos y esperamos intensificar. También, recientemente la Sociedad ha firmado una asociación estratégica con ALAT y ERS (European Respiratory Society) lo cual le permite variados beneficios a nuestros socios.

El año 2016 tendremos la posibilidad de participar como asistentes, o conferencistas en el $10^{\circ}$ Congreso ALAT que se realizará en Santiago de Chile, que representa toda una gran oportunidad de actualización en enfermedades respiratorias.

Por otro lado, no debemos cesar nuestra lucha contra el tabaquismo. El consumo de tabaco continúa siendo la principal causa de enfermedades y muertes prevenibles en todo el mundo.

Todos sabemos que nuestro país tiene una triste estadística sobre el consumo de tabaco. El $40,6 \%$ de la población mayor de 15 años fuma y los jóvenes chilenos son los más fumadores del mundo ${ }^{2}$. También, sabemos hoy en día que el tabaquismo es un factor de riesgo de EPOC, Cáncer pulmonar (que es la segunda causa de muerte en hombres y la tercera en mujeres), de neumonías, también de fibrosis pulmonar idiopática, sin contar las enfermedades cardiovasculares entre otros daños.

Por ello, debemos intensificar nuestros esfuerzos contra este hábito letal y la prevención debería ser nuestro lema. Esta, debe focalizarse en las poblaciones más jóvenes, promoviendo con más fuerza la consejería antitabaco a todo nivel y lograr tratamiento para todos aquellos que quieren dejar de fumar. Sin duda, que esto requiere una asociación estratégica con los Ministerios de Salud y de Educación para promover campañas de prevención en los colegios y a nivel de atención primaria, lo que permitiría multiplicar nuestros esfuerzos.

La Sociedad Chilena de Enfermedades Respiratorias viene trabajando desde hace varios años 
en contra del tabaquismo. La Organización Mundial de la Salud propició un tratado internacional de salud pública para el control de esta epidemia en el mundo, que culminó con la aprobación, en el año 2003, del Convenio Marco de Control del Tabaco. En Chile este tratado fue aprobado en marzo de 2005, gracias a un trabajo sistemático que realizó la comisión de tabaquismo de la Sociedad liderada por el Dr. Sergio Bello ${ }^{3}$.

Recientemente, se logró la modificación de la ley del tabaco (Ley $\mathrm{N}^{\circ}$ 20.660), un trabajo de la Sociedad Chilena de Enfermedades Respiratorias liderada en ese entonces por la Dra. Carolina Herrera, presidenta de la Sociedad y que demuestra que se puede lograr influir -en buena ley y con argumentos- en políticas y leyes públicas de la nación que tendrán alto impacto en la población.

Esta visión, puede quedarse en sólo anhelos y sueños. Para que ello sea realidad, se requiere: 1) Participación de diversos miembros de la Sociedad; 2) Recursos económicos necesarios para apoyar estas actividades. Es por ello que se creó recientemente la Fundación SER Chile, que deberá desarrollar las estrategias necesarias para obtener y proveer los recursos financieros para desarrollar las diversas actividades de la Sociedad; 3) Por otro lado, y más importante aún es lo que realizó la SEPAR en 2008, la ERS en 2010, o la ALAT en 2014 que fue definir su "Plan Estratégico" para los próximos años ${ }^{4}$. Esta labor no es sólo del presidente, ni del directorio sino de todos los socios para desarrollar esta ruta.

En mi opinión, la "visión" de la Sociedad Chilena de Enfermedades Respiratorias debe ser "liderar el conocimiento de las enfermedades respiratorias y torácicas en el país y gestionarlo de forma activa para convertirse en el referente clave y aliado imprescindible, de todos los agentes relacionados".

\section{Bibliografía}

1.- VÁZQUEZ-GARCÍA J C, SALAS-HERNÁNDEZ J, PÉREZ PADILLA R, MONTES DE OCA M. Salud respiratoria en América Latina: número de especialistas $\mathrm{y}$ formación de recursos humanos. Arch Bronconeumol 2014; 50: 34-9.

2.- VALDIVIA G. Encuesta nacional de salud 2009-2010: Enseñanzas y desafíos. Rev Chil Enferm Respir 2011; 27: 5-6.

3.- BELLO S. Tratamiento del Tabaquismo. Rev Chil Cardiol 2011; 30: 230-9.

4.- ANCOCHEA J. SEPAR-visión de la investigación. Arch Bronconeumol 2008; 44: 457-8.

Correspondencia a:

Dr. Francisco Arancibia H.

José M. Infante 717, 3er piso

Email: fearancibia@gmail.com 